\title{
Apport de l'imagerie SAR de la houle au génie côtier
}

\author{
Philippe Forget * et Antoine Mangin ${ }^{* *}$ \\ * LSEET CNRS, Université de Toulon et du Var, 83957 La Garde cedex \\ ${ }^{* *}$ ACRI, 06904 Sophia-Antipolis cedex
}

Résumé: On présente des exemples de produits dérivés d'images par radar à ouverture synthétique satellital de phénomènes de houle côtière à grande échelle et à l'échelle des transformations littorales. Les procédés de restitution des crêtes et hauteurs sont décrits et la cohérence physique des résultats est démontrée par la mise en oeuvre de modélisations. On met l'accent en conclusion sur les potentialités de ce procédé de télédétection active pour la modélisation.

\section{Introduction}

Le SAR est une technique déjà éprouvée pour l'observation des champs de vagues. Un grand nombre d'études ont été consacrées ces quinze dernières années aux mécanismes de l'imagerie et à leur représentation mathématique afin de pouvoir utiliser quantitativement les images radar des vagues et, essentiellement, pour inverser le spectre directionnel des vagues. Une avancée importante est due à Hasselmann et Hasselmann (1993) (HH) qui, les premiers, ont présenté à la fois la formulation de la transformation directe, non-linéaire, du spectre des vagues, et une méthode inverse adaptée à cette formulation. Cet ensemble conceptuel est destiné à un usage routinier des données satellitales de missions de type ERS 1/2, qui présentent un mode d'acquisition spécifique aux vagues du large destiné à en assurer une couverture globale. Ce type de données devrait améliorer les prédictions d'état de mer grâce, notamment, à la mise en oeuvre de techniques d'assimilation des spectres restitués par les modèles opérationnels. D'autres approches peuvent être envisagées pour analyser les images de houle dans la zone côtière. Après un rappel des principes de l'imagerie radar de la houle, nous présentons ici quelques exemples de «produits " issus d'images radar de houle, nécessitant un traitement minimal, donc rapide en temps de calcul, cohérent avec la théorie de l'imagerie, et susceptiules de présenter un certain intérêt pour le génie côtier.

\section{Principes de l'imagerie de la houle}

Ces principes sont connus depuis longtemps (e.g., Brüning et al., 1994): variations de l'incidence locale des ondes radar du fait des pentes de la houle, modulation hydrodynamique par la houle de la rúgosité de Bragg captée par le radar (vagues de longeur d'onde de centi- à déci-métriques), effet d'accumulation de diffuseurs phasée avec la houle et résultant d'effets Doppler consécutifs au traitement cohérent en synthèse d'ouverture. Ce dernier effet atteint un maximum d'efficacité pour une houle se propageant azimutalement (parallèlement à la direction de vol). Cependant, devenant rapidement non-linéaire quand la cambrure augmente, ce mécanisme est, en fait, le principal facteur limitatif de l'imagerie des vagues. Un dernier mécanisme est en oeuvre suivant le plan d'incidence (direction radiale) par 
un effet d'accumulation des diffuseurs lié à l'échantillonnage temporel des signaux parvenant d'une surface non plane. Les théories de l'imagerie des vagues peuvent s'exprimer en terme d'une fonction de transfert (FT), $Q(k)$, caractérisant la relation entre le spectre d'image et le spectre des vagues. Cette fonction résulte de la. sommation cohérente $T(k)$ des FT associées aux processus décrits (d'expression plus ou moins bien connue suivant le processus: ainsi, celle la FT hydrodynamique n'est qu'approchée), puis d'une transformation du résultat par des opérations mathématiques aboutissant à l'expression au premier ordre suivante:

$$
Q(k)=|T(k)|^{2} \exp -\left(k_{x} / k_{c}\right)^{2}
$$

La fonction exponentielle réalise une coupure azimutale dans le spectre de l'image, couramment observêe dans les cas réels (Figure 1). Les vagues de composante azimutale $k_{x}$ supérieure à $k_{c}$ ne sont pas imagées. La composante radiale $k_{y}$ étant, elle, préservée, le résultat net est une possible distorsion des longueurs d'onde et directions par rapport à leur vraie valeur. Ce phénomène n'affecte pas la houle, qui, dans le plan spectral, peut être assimilée à un Dirac de nombre d'onde $k_{H}$, à la condition que $k_{H}\left(\equiv\left|k_{H}\right|^{2}\right.$ ) soit inférieur à $k_{c}$. Dans le cas contraire, la houle est absente de l'image.

Forget et al. (1993) ont établi une méthode d'inversion de la hauteur significative $H$ de la houle dans le cas où $k_{c}$ ne dépend que de la houle elle-même. La méthode est légitime lorsque la mer du vent est faiblement énergétique, justifiant l'approximation constituée par la formule (1). Cette méthode a été étendue récemment (Vachon et al., 1994; Rousseau et Forget, 1997) au cas où une mer du vent est surimposée à la houle. Le procédé consiste à déterminer la largeur effective de la coupure spectrale, $k_{c}{ }^{\prime}$, puis à calculer la FT grâce à une expression identique à (1) mais utilisant une expression de $k_{c}$ fonction de $k_{c}^{\prime}$, cette fonction étant déterminée en utilisant le modèle complet de $\mathrm{HH}$ appliqué à une variété de spectres de vagues synthétiques. Le résultat est une augmentation des valeurs de $H$ par rapport à l'utilisation de la méthode précédente. Des estimations de $H$ sont données dans le Tableau 1 et comparées avec des mesures in situ, $H_{m e s}$. Notons que les valeurs calculées sont déduites de l'énergie du pic de houle de l'image, alors que les mesures in situ sont relatives à l'état de mer total, ce qui explique sans doute pour une grande part que $H_{m e s}$ soit supérieur à $H$.

\begin{tabular}{cccc}
\hline Date & H eq. (1) & H conige & H mesure \\
\hline 28.10 .96 & $2.0 \mathrm{~m}$ & 2.0 & 3.6 \\
22.01 .97 & 1.2 & 1.3 & 1.7 \\
10.02 .97 & 0.8 & 0.9 & 2.5 \\
14.03 .97 & 1.8 & 2.0 & 2.1 \\
17.03 .97 & 1.1 & 1.7 & 1.5 \\
\hline
\end{tabular}

Tableau 1: Estimations de la hauteur significative de la houle sur des images ERS2 dans la région de l'île d'Yeu et comparaisons avec des mesures de terrain.

En résumé, l'imagerie radar est en général capable de fournir une représentation fidèle des crêtes et de l'amplitude de la houle. Cependant, pour certaines 
conditions portant à la fois sur l'énergie de la mer du vent, qui régit dans une large mesure la coupure azimutale, et la direction des crêtes, l'information peut être perdue. Notons que, du fait des déphasages introduits par la FT d'imagerie, la position des crêtes n'est pas garantie à mieux qu'une longueur d'onde.

\section{Images de houle côtière à grande échelle}

Un des grands intérêts de l'imagerie radar satellitaire est la taille importante des zones côtières qu'elle peut couvrir. Ainsi, la taille d'une image ERS est de $100 \times 100 \mathrm{~km}^{2}$ (la résolution, de $30 \mathrm{~m}$, représente, par contre, un aspect limitant puisque, suivant le critère de Nyquist, seules les vagues de plus de $60 \mathrm{~m}$ sont effectivement détectées). Un premier produit dérivé d'images SAR est la cartographie du nombre d'onde et de la hauteur significative. Une fenêtre glissante parcourt la scène et y détermine des «imagettes » qui sont traitées suivant un certain protocole propre aux images SAR et consistant à calculer les spectre de modulation corrigés de l'influence du speckle et des éventuelles corrélations microscopiques des pixels, à détecter le pic de houle, à déterminer sa position $k$ et à inverser l'énergie du pic pour restituer la hauteur $H$. La figure 2.a résulte du traitement de 2 images ERS1 contiguës au large de la Mauritanie. La fenêtre d'analyse est de $6.4 \times 6.4 \mathrm{~km}^{2}$ Une houle de près de $300 \mathrm{~m}$ subit des déformations évidentes du nombre d'onde consécutives à la bathymétrie rencontrée. Ces déformations sont conformes aux simulations faites par la méthode classique des rayons (Fig. 2.b). Les hauteurs significatives (Fig. 2.c) apparaissent physiquement compatibles avec les observations de réfraction. Les valeurs de hauteur $(47 \mathrm{~cm})$ et de longueur d'onde $(289 \mathrm{~m})$ au large ont été validées par le modèle VAGATLA de Météofrance ( $34 \mathrm{~cm}$ et $259 \mathrm{~m}$ respectivement). Ce type d'observation à grande échelle des variations de la houle côtière illustre l'adéquation de la donnée radar pour la validation des modèles de propagation à cette échelle, et/ou la validation de la bathymétrie d'un site peu documenté ou à forte variabilité temporelle. De plus, les paramètres de houle déduits d'une image radar signent éventuellement la présence de courants (Forget et al, 1996).

\section{Images de houle côtière à petite échelle}

La houle subit les modifications les plus marquées près des côtes (caps, baies etc.) ou au voisinage des îles. Il est alors intéressant de concentrer l'analyse sur des petites portions d'images et d'envisager des produits plus élaborés que les pirécédents. Une difficulté à résoudre est le caractère souvent très inhomogène du champ de vagues, ce qui oblige à travailler sur des fenêtres d'analyse étroites pouvant ne comporter que quelques vagues. Des méthodes super-résolvantes (filtrage AR, maximum d'entropie etc.) ont déjà été mises en oeuvre sur des images radar pour résoudre cette difficulté (Cariou, 1991). Ce sont des méthodes lourdes et parfois délicates à appliquer, pour un résultat qui n'est pas toujours garanti. On retiendra deux méthodes fondées l'une sur l'analyse de Fourier et l'autre sur la transformée en ondelettes. Cès méthodes sont appliquées ici dans le but de restituer les crêtes de la houle, qu'un traitement aval (non réalisé ici) pourrait traduire en plan de houle. L'autre produit recherché est le champ détaillé des hauteurs significatives relatives, typiquement, à quelques longueurs d'onde. 


\subsection{Restitution des crêtes}

La première méthode consiste, comme en $\$ 3$, à parcourir la portion d'image sélectionnée au moyen d'une petite fenêtre, de taille suffisante cependant pour contenir quelques oscillations du signal de modulation, puis de filtrer l'imagette au voisinage du nombre d'onde du pic (filtre passe-bande exponentiel). Afin d'éviter les effets de bord, seule la partie de l'imagette filtrée à l'intersection de deux positions de la fenêtre, se chevauchant typiquement sur une demi-aire, est conservée. L'expérience prouve que l'image originale doit avoir été préalablement filtrée par un filtre passe-bande atténuant le bruit de granulométrie, puis avoir été «expertisée » de manière à ce que les pics détectés soient physiques. Cette opération est réalisée dans le cas présent par l'examen de l'histogramme des nombres d'onde calculés lors d'une première exploration de l'image. L'image des crêtes est obtenue en binarisant les imagettes filtrées. Le procédé est illustré sur la figure 3.a relative à une portion de l'image de la figure 2 . On note quelques crêtes aberrantes. Les zones vierges correspondent à une absence de signal détectable sur l'image d'origine du fait, soit de ia Iongueur finie des crêtes (cohérence latérale des crêtes limitée), soit d'effet locaux d'affaiblissement de l'amplitude locale. L'algorithme pourrait être amélioré par un algorithme de raccordement des crêtes, qui compenserait l'absence d'imagerie au niveau de ces minima d'amplitude. Les transformations de la houle à l'approche de la côte sont particulièrement nettes. Un autre exemple est montré dans la région de l'île d'Yeu (28.10.96, Fig. 4.a). Là encore, des figures de réfraction-diffraction sont clairement détectées d'après la distribution des crêtes, malgré des défauts dus à des mauvaises détections ou bien à des rapports signal à bruit insuffisants.

L'autre méthode consiste dans une procédure de filtrage par applications successives d'une transformée en ondelette. Un algorithme permet d'évaluer pas à pas les paramètres courants de l'ondelette analysante (taille et localisation) permettant de restituer les contributions des différentes longueurs d'onde du signal initial sans recouvrement. La méthode se résume, très schématiquement, à l'application d'un traitement pas à pas du signal par un filtre passe-bas dont la fréquence de coupure varie à chaque pas. Un choix judicieux du filtre (ici, une spline cubique) permet d'exprimer chaque niveau filtré en fonction du précédent. Un prétraitement de l'image originale (filtrage directionnel) est nécessaire pour rendre compte des obliquités de la houle. Le procédé est appliqué à des imagettes analysantes de $64 \times 64$ pixels. Un exemple de restitution des crêtes est montré sur la figure 5 (île d'Yeu, 14.03.97). On voit, en particulier, que le traitement fait apparaître des croisements de houles à l'est de l'île. Des transformations côtières évidentes de la houle dans le golfe de Giens (Méditerranée) apparaissent sur la figure 6.a (Lajoie et Mangin, 1995). La modélisation de l'équation de Berkhoff parabolisée, mise en oeuvre ici pour une onde mono-chromatique (Lajoie, 1996), permet de restituer les effets combinés de réfraction/diffraction (Fig. 6.b).

\subsection{Restitution des hauteurs}

Concernant l'image de la figure 3, les valeurs calculées de $H$ (Fig. 3.c), sont de l'ordre de $50 \mathrm{~cm}$ au large et peuvent atteindre $3 \mathrm{~m}$ près de la côte. On note l'aspect hétérogène du champ des hauteurs. Au large, cet aspect est davantage en liaison, 
semble t-il, avec la structure en «groupe» des lames de houle (modulation spatiale de l'enveloppe) que du fait de transformations côtières. Le traitement d'image par transformée de Hilbert est une autre méthode permettant l'identification de cette propriété morphologique des vaguès longues, bien connue et très couramment observée sur les images radar (Forget, 1996). La mise en oeuvre du modèle de rayons (Fig. 3.b), les rayons étant initialisés par les nombres d'onde déterminés sur l'image, ne restitue qu'une seule des zones d'intensification de la hauteur significative le long de la côte (au nord). A l'inverse, la concentration des rayons calculés au sud de l'image ne donne pas lieu à une augmentation significative de $H$ sur l'image. L'échantillonnage trop rudimentaire des profondeurs (provenant d'une carte SHOM) est sans doute à l'origine de ces différences. L'image de l'île d'Yeu (hauteurs lissées et interpolées Fig. 4.c) conduit à des observations analogues. Des valeurs atteignant $5 \mathrm{~m}$ sont trouvées au niveau des zones brillantes, pour une valeur moyenne comprise entre 2 et $3 \mathrm{~m}$ sur le bord ouest de l'image. En outre, on remarque au nord de l'île une diminution marquée de $H$ dans la région de divergence des rayons et une augmentation (point brillant) au point de convergence (Fig. $4 . \mathrm{b}$ ).

L'effet de coupure azimutale $(\$ 2)$ n'a pas été pris en compte dans les estimations des figures 3 et 4 (la très petite taille de la fenêtre d'analyse ne permettant pas d'évaluer précisément le paramètre $\lambda_{c}$ ). Un aménagement du code est en cours afin d'appliquer localement aux estimations de $H$ les corrections de coupure azimutale déduites d'une première exploration de l'image à l'aide d'une fenêtre large (ces corrections varient de 1 à 2 dans le cas de la figure 4).

\section{Conclusions}

Il est banal de dire que les modèles de vagues côtières souffrent d'un manque de données réelles, qui soient, en particulier, adaptées à leur emprise d'espace (ainsi, une bouée de houle donne une description ponctuelle de l'état de mer). Seule la donnée de télédétection noùs parait pertinente de ce point de vue (la variabilité temporelle n'étant pas, quant à elle, accessible à ce procédé) et, spécialement, l'imagerie SAR satellitale, capable de représenter spatialement les crêtes et les amplitudes locales de la houle (l'imagerie visible, e.g. SPOT, reste problématique poui la restitution des amplitudes). La perception d'une houle par imagerie radar est originale par rapport à la modélisation: groupage des vagues, crêtes de longueur finie, détails. En conclusion, on peut souhaiter un usage plus répandu qu'il ne l'est des produits de télédétection radar pour tester, valider et valoriser les travaux de modélisation des vagues en zone côtière.

Ce travail a été réalisé dans le cadre du programme AVALSAR (CNES), contrat 96/CNES/0380, et avec le soutien de l'Agence Spatiale Européenne.

\section{Références}

Brïning, C., Schmidt, R. and W. Alpers, 1994: Estimation of the ocean waveradar modulation transfer function from synthetic aperture radar imagery. $J$. Geophys.Res.,99, 9803-9815.

Cariou, C., 1991: Analyse spectrale d'images numériques, application à la télédétection spatiale des océans. Thèse Université de Bretagne Occidentale.

Forget, P., 1994: Monitoring of swell by ERS1 synthetic aperture radar. 2nd ERS1 Workshop Proceedings., Brest, 15-17 mars 1994, 60-65. 
Forget, P., Broche, P. and F. Cuq, 1995: Principles of swell mesaurements by SAR with application to ERS1 observations off the Mauritanian coast. Int. J. Remote Sensing, 16, 2413-2422.

Hasselmann, K. and S. Hasselmann, 1991: On the nonlinear mapping of an ocean wave spectrum into a synthetic aperture radar image spectrum and its inversion. J. Geophys. Res., 96, 10713-10729.

Lajoie, D., et A. Mangin, 1995: Diffraction réfraction de la houle : couplage avec un modèle d'agitation portuaire et comparaison avec des mesures satellitales. Communication Vèmes Journées de l'Hydrodynamique, Rouen, Mars 1995

Lajoie, D: 1996: Modélisation de la houle en zone côtière : prévision de l'agitation à l'intérieur des ports et mise au point d'atténuateurs de houle dynamiques. Thèse Universite d'Aix-Marseille II .

Vachon, P.W., Krogstad, H.E. and J.S. Paterson, 1994: Airborne and spaceborne synthetic aperture radar observations of ocean waves. Atmophere-Oceans, 32, 83-112.

Rousseau, S. and P. Forget, 1997: Significant wave height and wave imaging transfer function estimations from coastal SAR images in storm conditions. Proc. of the 3rd Airborne Remote Sensing Conf. and Exhibition, Copenhagen, 7-10 July 1997, 11.93-11-99.

\section{Figures}

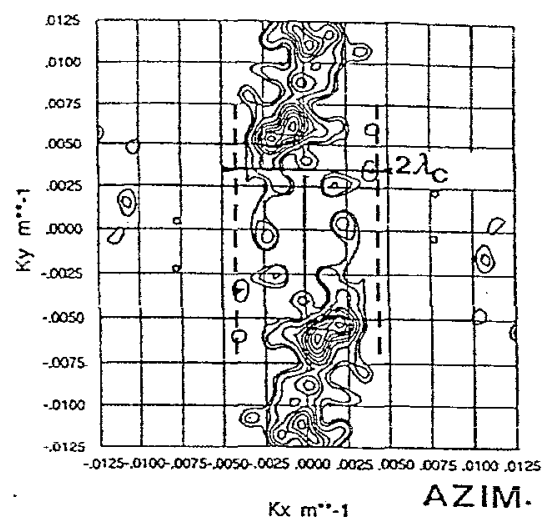

Fig. 1: Spectre de modulation SAR par situation de tempête (ERS1, région de la baie d'Audierne).

(a)

(b)

(c)
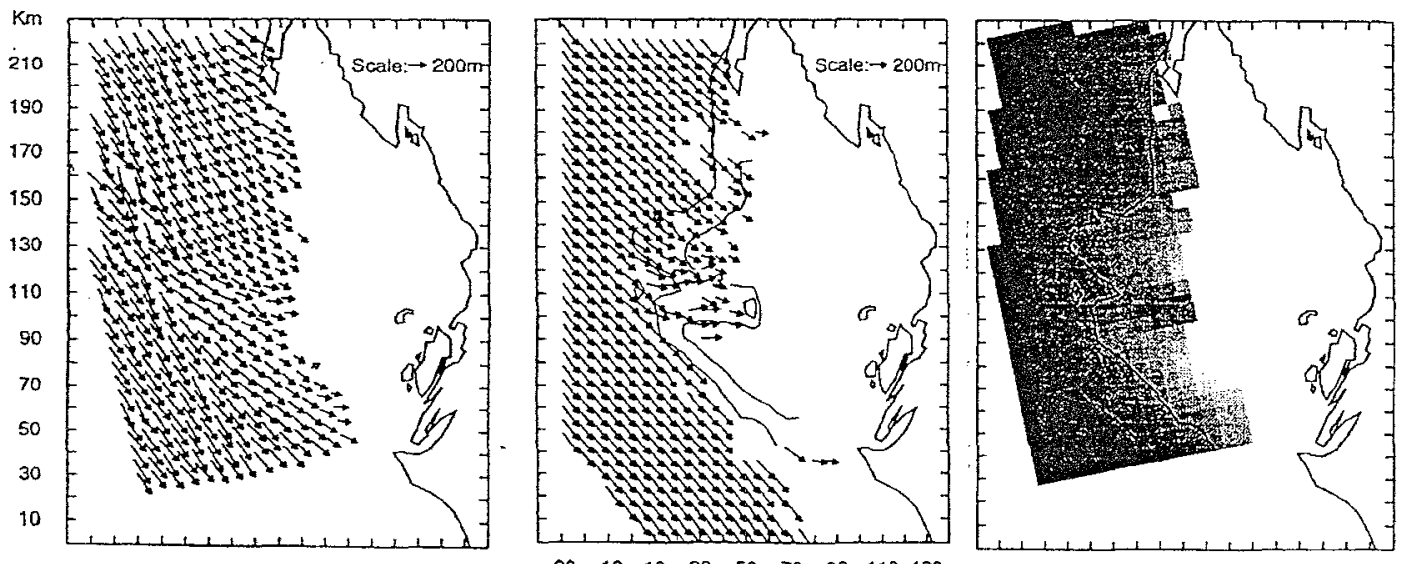

Fig. 2: Champ des longueurs d'onde-directions déduit de 2 images ERS1 (a) et d'une simulation (b). Champ des hauteurs significatives (c) (Mauritanie). 
(a)

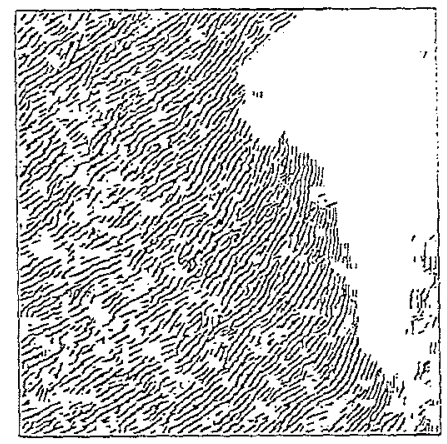

(c)

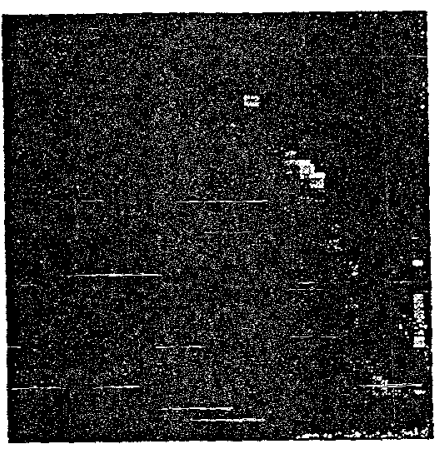

(a)

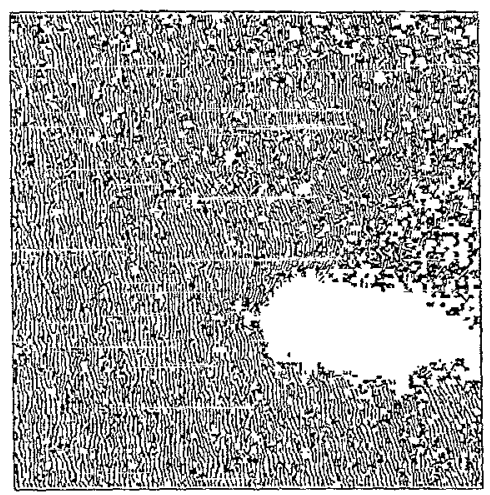

(c)

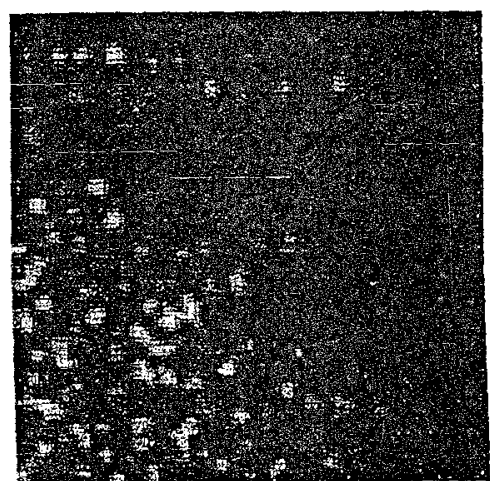

(b)

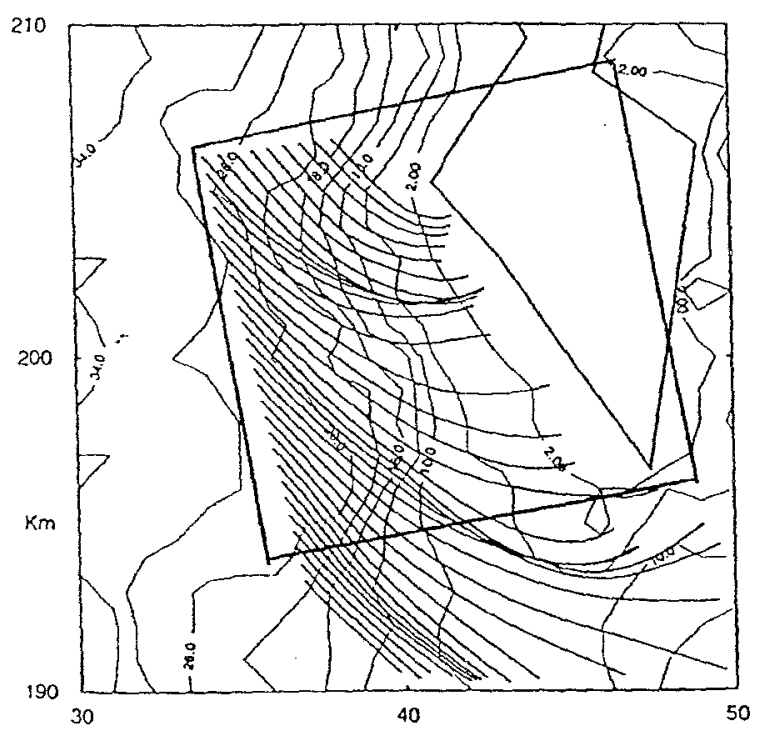

Fig. 3: Crêtes de houle (a), simulation des rayons de houle (b) et champ des hauteurs $(c)$ sur une portion de $\mathrm{l}_{2}$ image de la Fig. $2\left(12.8 \times 12.8 \mathrm{~km}^{2}\right)$ (b)

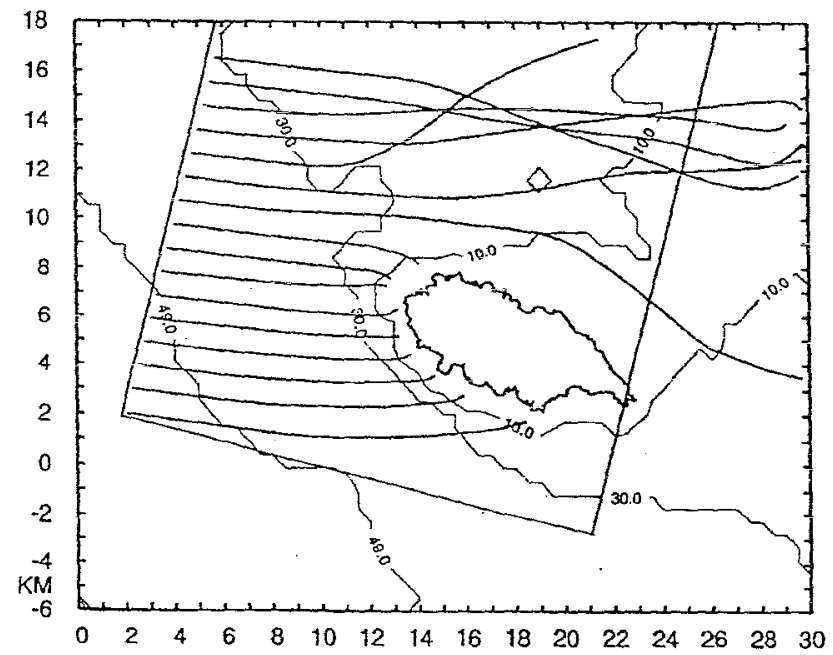

Fig. 4: Crêtes de houle (a), simulation des rayons de houle (b) et champ des hauteurs (c) calculés sur une portion de l'image ERS2 del' île d'Yêu du 28.10 .96 ( taille: $20 \times 20 \mathrm{~km}^{2}$ ). 


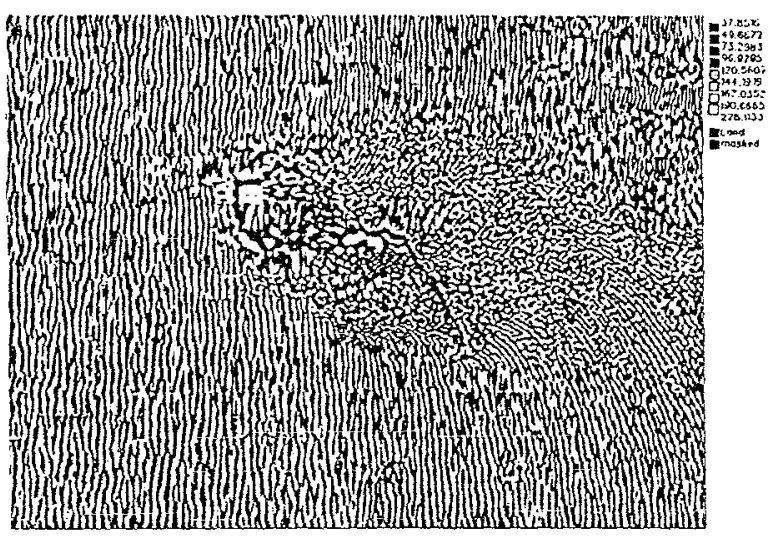

Fig. 5: Résultat de l'analyse par ondelettes d'une portion de l'image ERS2 de l'île d'Yeu du 14.03.97 ( taille: $24 \times 17.6 \mathrm{~km}^{2}$ )

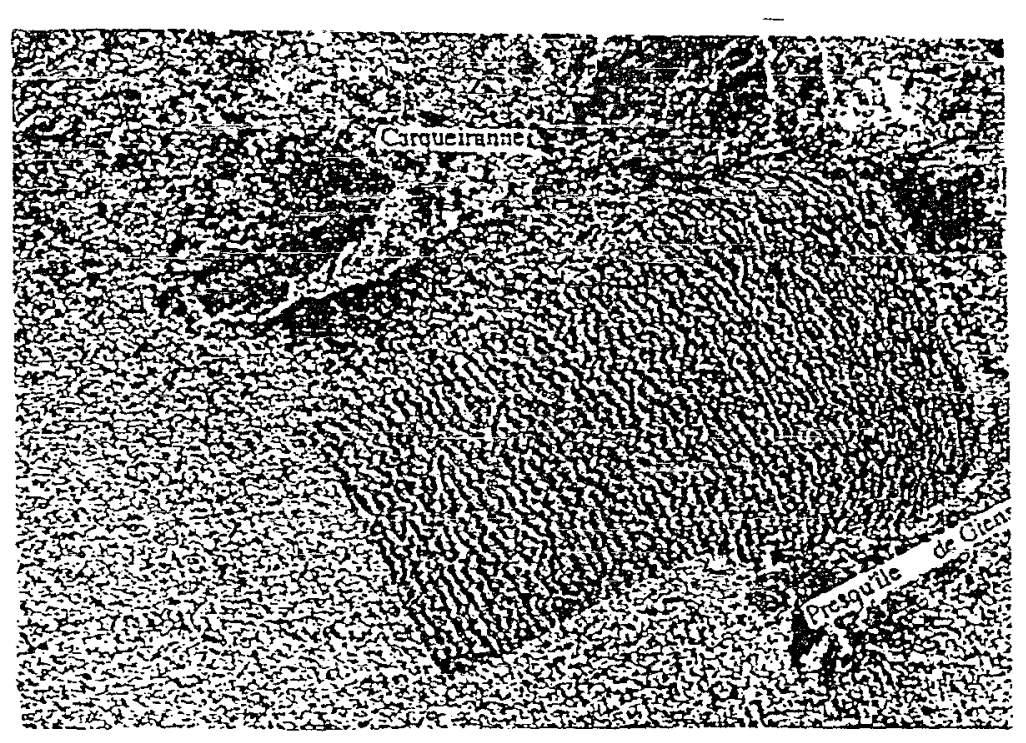

(a)

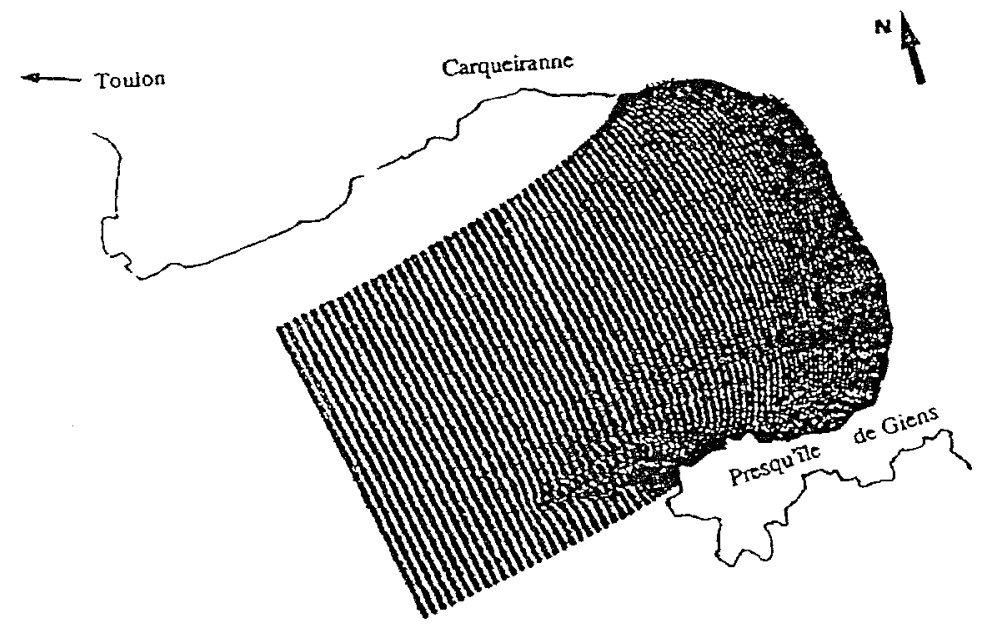

(b)

Fig. 6: Traitement en ondelettes d'une image ERS1 dans le golfe de Giens (a) (02.04.93, taille: $9.5 \times 11 \mathrm{~km} 2$ ) et modélisation par l'équation de Berkhoff (b). 